\title{
ARTICLE
}

\section{Hybrid integrated biological-solid-state system powered with adenosine triphosphate}

Jared M. Roseman ${ }^{1}$, Jianxun Lin ${ }^{1}$, Siddharth Ramakrishnan², Jacob K. Rosenstein ${ }^{3}$ \& Kenneth L. Shepard ${ }^{1}$

There is enormous potential in combining the capabilities of the biological and the solid state to create hybrid engineered systems. While there have been recent efforts to harness power from naturally occurring potentials in living systems in plants and animals to power complementary metal-oxide-semiconductor integrated circuits, here we report the first successful effort to isolate the energetics of an electrogenic ion pump in an engineered in vitro environment to power such an artificial system. An integrated circuit is powered by adenosine triphosphate through the action of $\mathrm{Na}^{+} / \mathrm{K}^{+}$adenosine triphosphatases in an integrated in vitro lipid bilayer membrane. The ion pumps (active in the membrane at numbers exceeding $2 \times 10^{6} \mathrm{~mm}^{-2}$ ) are able to sustain a short-circuit current of $32.6 \mathrm{pA} \mathrm{mm}^{-2}$ and an

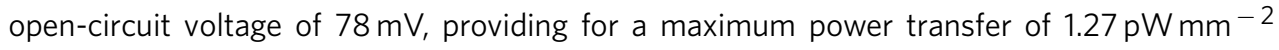
from a single bilayer. Two series-stacked bilayers provide a voltage sufficient to operate an integrated circuit with a conversion efficiency of chemical to electrical energy of $14.9 \%$.

\footnotetext{
${ }^{1}$ Department of Electrical Engineering, Columbia University, New York, New York 10027, USA. ${ }^{2}$ Department of Biology, Neuroscience Program, University of Puget Sound, Tacoma, Washington 98416, USA. ${ }^{3}$ School of Engineering, Brown University, Providence, Rhode Island 02912, USA. Correspondence and requests for materials should be addressed to K.L.S. (email: shepard@ee.columbia.edu).
} 
T he energetics of living systems are based on electrochemical membrane potentials that are present in cell plasma membranes, the inner membrane of mitochondria, or the thylakoid membrane of chloroplasts ${ }^{1}$. In the latter two cases, the specific membrane potential is known as the proton-motive force and is used by proton adenosine triphosphate (ATP) synthases to produce ATP. In the former case, $\mathrm{Na}^{+} / \mathrm{K}^{+}$. ATPases hydrolyse ATP to maintain the resting potential in most cells.

While there have been recent efforts to harness power from some naturally occurring potentials in living systems that are the result of ion pump action both in plants ${ }^{2}$ and animals 3 , to power complementary metal-oxide semiconductor (CMOS) integrated circuits (ICs), this work is the first successful effort to isolate the energetics of an electrogenic ion pump in an engineered in vitro environment to power such an artificial system. Prior efforts to harness power from in vitro membrane systems incorporating ionpumping ATPases ${ }^{5-9}$ and light-activated bacteriorhodopsin ${ }^{9-11}$ have been limited by difficulty in incorporating these proteins in sufficient quantity to attain measurable current and in achieving sufficiently large membrane resistances to harness these currents. Both problems are solved in this effort to power an IC from ATP in an in vitro environment. The resulting measurements provide new insight into a generalized circuit model, which allows us to determine the conditions to maximize the efficiency of harnessing chemical energy through the action of electrogenic ion pumps.

\section{Results}

ATP-powered IC. Figure 1a shows the complete hybrid integrated system, consisting of a CMOS IC packaged with an ATPharvesting 'biocell'. The biocell consists of two series-stacked ATPase bearing suspended lipid bilayers with a fluid chamber directly on top of the IC. Series stacking of two membranes is necessary to provide the required start-up voltage for IC and eliminates the need for an external energy source, which is typically required to start circuits from low-voltage supplies ${ }^{2,3}$. As shown in Fig. 1c, a matching network in the form of a switched capacitor allows the load resistance of the IC to be matched to that presented by the biocell. In principle, the switch $S$ can be implicit. The biocell charges $C_{\mathrm{STOR}}$ until the self start-up voltage, $V_{\text {start }}$ is reached. The chip then operates until the biocell voltage drops below the minimum supply voltage for operation, $V_{\text {min }}$. Active current draw from the IC stops at this point, allowing the charge to build up again on $C_{\mathrm{STOR}}$. In our case, however, the IC leakage current exceeds $13.5 \mathrm{nA}$ at $V_{\text {start }}$, more than can be provided by the biocell. As a result, an explicit transistor switch and comparator (outside of the IC) are used for this function in the experimental results presented here, which are not powered by the biocell and not included in energy efficiency calculations (see Supplementary Discussion for additional details). The energy from the biocell is used to operate a voltage converter (voltage doubler) and some simple inverter-based ring oscillators in the IC, which receive power from no other sources.

A silver/silver-chloride $(\mathrm{Ag} / \mathrm{AgCl})$ microfabricated thin film on the surface of the chip, and an $\mathrm{Ag} / \mathrm{AgCl}$ pellet serve as the working (WE) and counter (CE) electrode to convert ions to electrons. Careful attention must be paid to the electrodes in this power-transfer application to ensure that the electrodes are not an uncontrolled galvanic energy source. The lipid (purified 1,2-dioleoyl-sn-glycero-3-phosphocholine, DOPC) bilayers are formed in a $250-\mu \mathrm{m}$ pore ${ }^{12}$ epoxied to a teflon housing (see Methods for details). Purified $5^{\prime}$-sodium potassium adenosine triphosphatase from porcine cerebral cortex is embedded into each bilayer (see Methods for details). Prior to the addition of ATP, the membrane produces no electrical power and has an $R_{\mathrm{m}}$ of $280 \mathrm{G} \Omega$. A 1.7-pA short-circuit (SC) current (Fig. 2b) through the membrane is observed upon the addition of ATP (final concentration $3 \mathrm{mM}$ ) to the cis chamber where functional, properly oriented enzymes generate a net electrogenic pump current. To perform these measurements, currents through each membrane of the biocell are measured using a voltage-clamp amplifier (inset of Fig. 2b) with a gain of $500 \mathrm{G} \Omega$ with special efforts taken to compensate amplifier leakage currents. Each ATPase transports three $\mathrm{Na}^{+}$ions from the cis chamber to the trans chamber and two $\mathrm{K}^{+}$ions from the trans chamber to the cis chamber (a net charge movement of one cation) for every molecule of ATP hydrolysed. At a rate of 100 hydrolysis events per second under zero electrical (SC) bias ${ }^{13}$, this results in an electrogenic current of $\sim 16 \mathrm{aA}$. The observed SC current corresponds to about $10^{5}$ active ATPases in the membrane or a concentration of about $2 \times 10^{6} \mathrm{~mm}^{-2}$, about $5 \%$ of the density of channels occurring naturally in mammalian nerve fibres ${ }^{14}$. It is expected that half of the channels inserted are inactive because they are oriented incorrectly.

Current-voltage characteristics of the ATPases. Figure 2a shows the complete measured current-voltage $(I-V)$ characteristic of a single ATPase-bearing membrane in the presence of ATP. The current due to membrane leakage through $R_{\mathrm{m}}$ is subtracted in the post-ATP curve. The $I-V$ characteristic fits a Boltzmann sigmoid curve, consistent with sodium-potassium pump currents measured on membrane patches at similar buffer conditions ${ }^{13,15,16}$. This nonlinear behaviour reflects the fact that the full ATPase transport cycle (three $\mathrm{Na}^{+}$ions from cis to trans and two $\mathrm{K}^{+}$ions from trans to cis) time increases (the turn-over rate, $k_{\mathrm{ATP}}$, decreases) as the membrane potential increases ${ }^{16}$. No effect on pump current is expected from any ion concentration gradients produced by the action of the ATPases (see Supplementary Discussion). Using this Boltzmann fit, we can model the biocell as a nonlinear voltagecontrolled current source $I_{\text {ATPase }}$ (inset Fig. 2a), in which the current produced by this source varies as a function of $V_{\mathrm{m}}$. In the fourth quadrant, where the cell is producing electrical power, this model can be linearized as a Norton equivalent circuit, consisting of a DC current source $\left(I_{\mathrm{p}}\right)$ in parallel with a currentlimiting resistor $\left(R_{\mathrm{p}}\right)$, which acts to limit the current delivered to the load at increasing bias $\left(I_{\mathrm{ATPa} e} \sim I_{\mathrm{p}}-V_{\mathrm{m}} / R_{\mathrm{p}}\right)$. Figure $2 \mathrm{c}$ shows the measured and simulated charging of $C_{\mathrm{m}}$ for a single membrane (open-circuited voltage). A custom amplifier with input resistance $R_{\text {in }}>10 \mathrm{~T} \Omega$ was required for this measurement (see Electrical Measurement Methods).

Reconciling operating voltage differences. The electrical characteristics of biological systems and solid-state systems are mismatched in their operating voltages. The minimum operating voltage of solid-state systems is determined by the need for transistors to modulate a Maxwell-Boltzmann (MB) distribution of carriers by several orders of magnitude through the application of a potential that is several multiples of $k T / q$ (where $k$ is Boltzmann's constant, $T$ is the temperature in degrees Kelvin and $q$ is the elementary charge). Biological systems, while operating under the same $\mathrm{MB}$ statistics, have no such constraints for operating ion channels since they are controlled by mechanical (or other conformational) processes rather than through modulation of a potential barrier. To bridge this operating voltage mismatch, the circuit includes a switched-capacitor voltage doubler (Fig. 1d) that is capable of self-startup from voltages as low $V_{\text {start }}=145 \mathrm{mV} \quad(\sim 5.5 \mathrm{kT} / \mathrm{q})$ and can be operated continuously from input voltages from as low as $V_{\min }=110 \mathrm{mV}$ (see Supplementary Discussion). In the series-stacked biocell with each membrane having approximately equal resistance and 

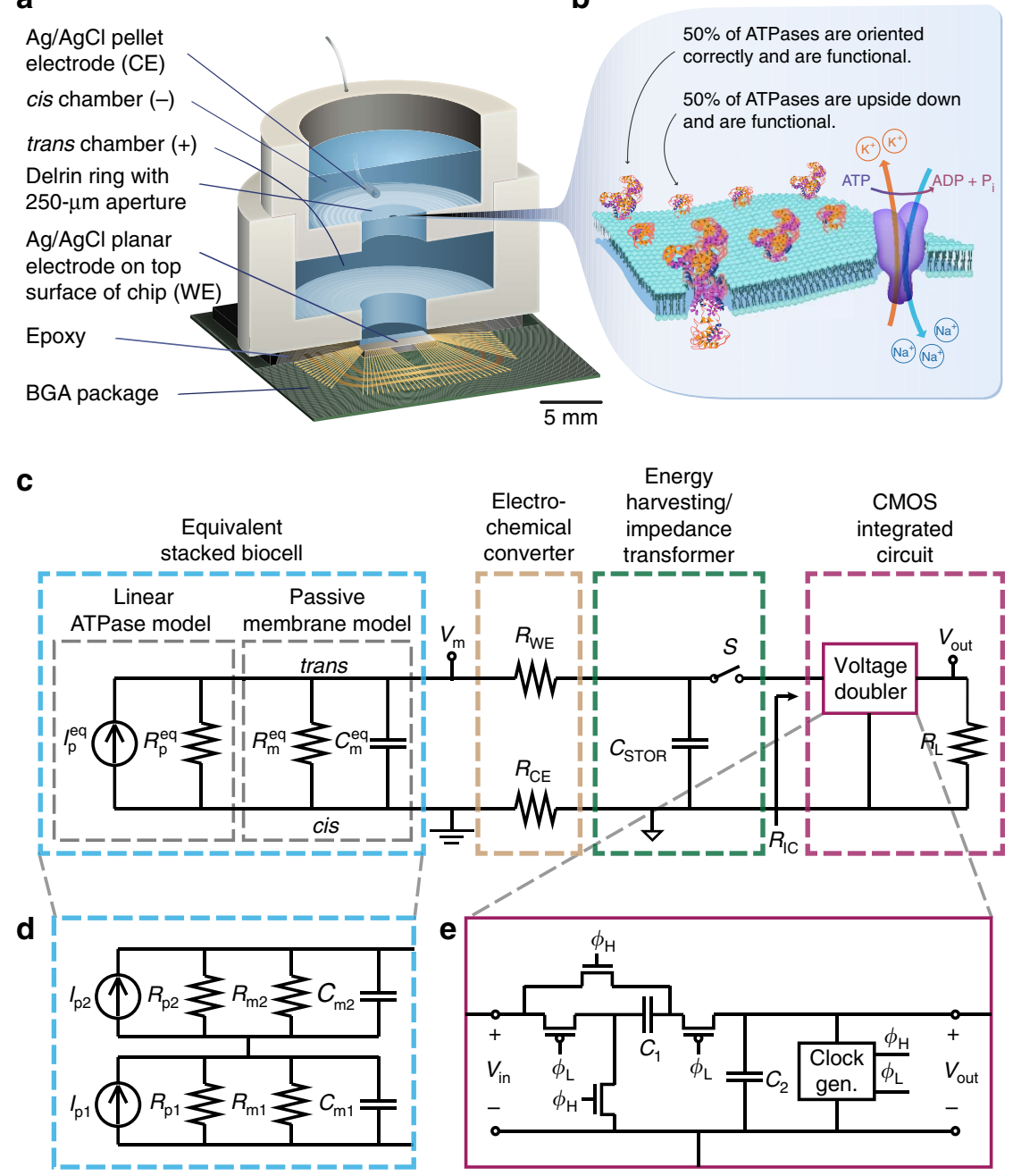

Figure 1 | Fully hybrid biological-solid-state system. (a) Illustration depicting biocell attached to CMOS integrated circuit. (b) Illustration of membrane in pore containing sodium-potassium pumps. (c) Circuit model of equivalent stacked membranes, leq $\left.\right|_{\mathrm{p}} ^{\mathrm{eq}}=2.1 \mathrm{pA}, R_{\mathrm{p}}^{\mathrm{eq}}=98.6 \mathrm{G} \Omega, R_{\mathrm{m}}^{\mathrm{eq}}=575 \mathrm{G} \Omega$ and $C_{\mathrm{m}}^{\mathrm{eq}}=75 \mathrm{pF}$, $\mathrm{Ag} / \mathrm{AgCl}$ electrode equivalent resistance $R_{\mathrm{WE}}+R_{\mathrm{CE}}<20 \mathrm{k} \Omega$, energy-harvesting capacitor $C_{\mathrm{STOR}}=100 \mathrm{nF}$ combined with switch as an impedance transformation network (only one switch necessary due to small duty cycle), and CMOS IC voltage doubler and resistor representing digital switching load. $R_{\mathrm{L}}$ represents the four independent ring oscillator loads. (d) Equivalent circuit detail of stacked biocell. (e) Switched-capacitor voltage doubler circuit schematic.

capacitance, $R_{\mathrm{m}}^{\mathrm{eq}}=2 R_{\mathrm{m}}$ and $C_{\mathrm{m}}^{\mathrm{eq}}=1 / 2 C_{\mathrm{m}}$. Once the converter has started up, operation can be maintained down to $110 \mathrm{mV}$, which means that each membrane in the two-stacked configuration can be biased to operate at potentials as low as $55 \mathrm{mV}$.

Maximizing the efficiency of harvesting energy from ATP. Solid-state systems and biological systems are also mismatched in their operating impedances. In our case, the biocell presents a source impedance, $R_{\mathrm{p}}^{\mathrm{eq}} \| R_{\mathrm{m}}^{\mathrm{eq}}=R_{\mathrm{p}}^{\mathrm{eq}} R_{\mathrm{m}}^{\mathrm{eq}} /\left(R_{\mathrm{p}}^{\mathrm{eq}}+R_{\mathrm{m}}^{\mathrm{eq}}\right)=84.2 \mathrm{G} \Omega$, while the load impedance presented by the complete integrated circuit (including both the voltage converter and ring oscillator loads) is approximately $R_{\mathrm{IC}}=200 \mathrm{k} \Omega$. (The load impedance, $R_{\mathrm{L}}$, of the ring oscillators alone is $305 \mathrm{k} \Omega$.) This mismatch in source and load impedance is manifest in large differences in power densities. In general, integrated circuits, even when operated at the point of minimum energy in subthreshold, consume on the order of $10^{-2} \mathrm{~W} \mathrm{~mm}^{-2}$ (or assuming a typical silicon chip thickness of $250 \mu \mathrm{m}, 4 \times 10^{-2} \mathrm{~W} \mathrm{~mm}^{-3}$ ) (ref. 17). Typical cells, in contrast, consume on the order of $4 \times 10^{-6} \mathrm{~W} \mathrm{~mm}^{-3}$ (ref. 18). In our case, a typical active power dissipation for our circuit is $92.3 \mathrm{nW}$, and the active average harvesting power is $71.4 \mathrm{fW}$ for the biocell. This discrepancy is managed through duty-cycled operation of the IC in which the circuit is largely disabled for long periods of time $\left(T_{\text {charge }}\right)$, integrating up the power onto a storage capacitor $\left(C_{\mathrm{STOR}}\right)$, which is then expended in a very brief period of activity $\left(T_{\text {run }}\right)$, as shown in Fig. 3a.

The overall efficiency of the system in converting chemical energy to the energy consumed in the load ring oscillator $(\eta)$ is given by the product of the conversion efficiency of the voltage doubler $\left(\eta_{\text {converter }}\right)$ and the conversion efficiency of chemical energy to electrical energy in the biocell $\left(\eta_{\text {biocell }}\right), \eta=\eta_{\text {converter }} \times$ $\eta_{\text {biocell }} \cdot \eta_{\text {converter }}$ is relatively constant over the range of input voltages at $\sim 59 \%$, as determined by various loading test circuits included in the chip design (Supplementary Figs 1-6). $\eta_{\text {biocell }}$, however, varies with transmembrane potential $V_{\mathrm{m}} \cdot \eta$ is the efficiency in transferring power to the power ring oscillator loads from the ATP harvested by biocell.

As reflected in the single-membrane circuit model of Fig. 4a, $V_{\mathrm{m}}$ in cells is the result of two related potentials, both derived from the action of the ion pumps: the potential that develops as a result of ionic concentration gradients of membrane permeable ions (the Nernst, or diffusion, potential, $V_{\text {diff }}$ ) and Ohmic potentials $\left(V_{\mathrm{p}}\right)$ due to the net electrogenic ionic currents of the ion pumps. These combine to determine the membrane potential, 

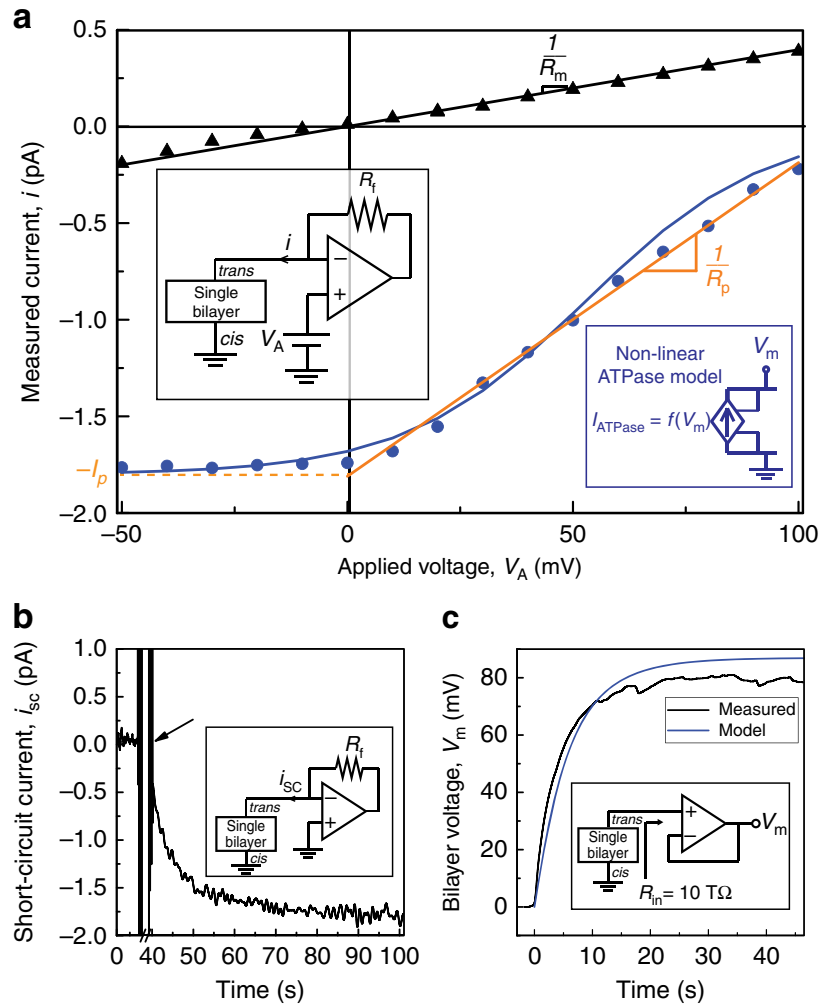

Figure 2 | Single-cell biocell characterization. (a) I-V sweeps of biocell before ( $\mathbf{\Delta})$ and after $(\mathbf{O})$ addition of ATP (3 mM final concentration). Voltages are stepped for this measurement. Bilayer current is the average of the last $5 \mathrm{~s}$ of a 10-s step (after charging currents have decayed away). Bilayer capacitance $C_{m}=153 \mathrm{pF}$ (consistent with DOPC bilayers ${ }^{12,30}$ ). PreATP data linear fit (black line) slope yield $R_{\mathrm{m}}=280 \mathrm{G} \Omega$. Post ATP data fit to a Boltzmann curve, slope $=0.02 \mathrm{~V}$ (blue line). Post-ATP linear fit (red line) yields $I_{\mathrm{p}}=-1.8 \mathrm{pA}$ and $R_{\mathrm{p}}=61.6 \mathrm{G} \Omega$, which corresponds to a per-ATP source resistance of $6.16 \times 10^{15}$. The current due to membrane leakage through $R_{-}\{m\}$ is subtracted in the post-ATP curve. (b) Short circuit current magnitude increasing to $1.7 \mathrm{pA}$ after the addition of $3 \mathrm{mM}$ ATP.

Transimpedance amplifier gain is $500 \mathrm{G} \Omega$. The headstage filter consists of a three-pole analog low-pass Bessel filter $f_{c}=10 \mathrm{kHz}$ in series with four-pole analog low-pass Bessel filter $f_{c}=100 \mathrm{~Hz}$. Data is post-processed with a lowpass digital filter with $f_{\mathrm{c}}=1 \mathrm{~Hz}$. (c) Biocell charging its own capacitance to $78 \mathrm{mV}$. Measured (black line). Simulated using non-linear model fit (blue line).

$V_{\mathrm{m}}=V_{\text {diff }}+V_{\mathrm{p}} . V_{\text {diff }}$ is determined by the superposition of the Thevenin 'Nernst' sources for each ionic species, $V_{\mathrm{Na}^{+}}, V_{\mathrm{K}^{+}}$, $V_{\mathrm{Mg}^{2+}}, \quad V_{\mathrm{Cl}^{-}}$, where $V_{X}=\frac{R T}{z F} \ln \left([X]_{\text {out }} /[X]_{\text {in }}\right), \quad R$ is the gas constant, $z$ is the valence of the species, $F$ is Faraday's constant and $[X]_{y}$ is the concentration of species $X$ in chamber $y . \mathrm{A}$ corresponding series resistance $R_{X}$ models the membrane permeability of those ions present in the system. An equivalent expression for $V_{\text {diff }}$ is also given by the Goldman-Hodgkin-Katz (GHK) equation. The total membrane resistance $R_{\mathrm{m}}$ is given by the parallel combination of $R_{\mathrm{Na}^{+}}, R_{\mathrm{K}^{+}}, R_{\mathrm{Mg}^{2+}}, R_{\mathrm{Cl}^{-}}$. Using the linear ATPase model (Fig. 1c), the electrogenic component of the transmembrane potential is given by $V_{\mathrm{p}}=I_{\mathrm{p}}\left(R_{\mathrm{p}} \| R_{\mathrm{m}}\right)$. Under physiological conditions, $V_{\mathrm{p}}$ is often negligible compared with $V_{\text {diff }}$ as a result of the relatively low value of $R_{\mathrm{m}}$ due to the presence of many transmembrane ion channels.

To first order, the energy made available to the $\mathrm{Na}^{+} / \mathrm{K}^{+}$ATPase by the hydrolysis of ATP is independent of the chemical or electric potential of the membrane and is given by $\left|\Delta G_{\mathrm{ATP}}\right| /$ $\left(q N_{\mathrm{A}}\right)$, where $\Delta G_{\mathrm{ATP}}$ is the Gibbs free energy change due to the

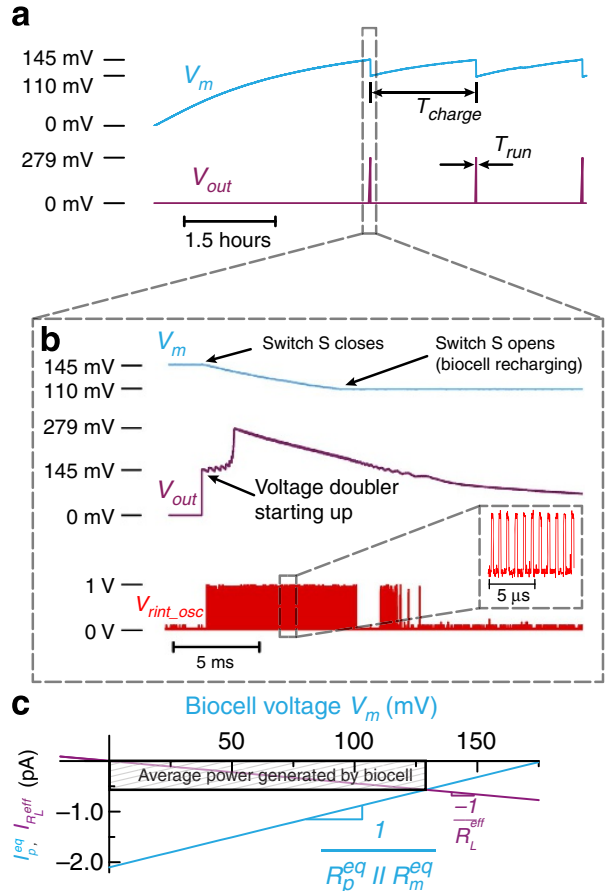

Figure 3 | Complete hybrid biological-solid-state system characterization. (a) Duty-cycled operation of the stacked biocell and CMOS IC. Shown are the input $\left(V_{m}\right)$ and output $\left(V_{\text {out }}\right)$ voltage of the voltage doubler. $T_{\text {charge }}=6250 \mathrm{~s}, T_{\text {run }}=5.5 \mathrm{~ms}$. During $T_{\text {run, }} 54.4 \mathrm{nW}$ delivered to the load and $37.9 \mathrm{nW}$ is consumed in running the voltage doubler. (b) Expanded waveform showing $V_{m}$ and $V_{\text {out }}$ near a switching transition and one of four ring oscillators. Inset shows the output of ring oscillator digital switching load switching at $1 \mathrm{MHz}$. (c) Biocell currentvoltage characteristic and effective load line.

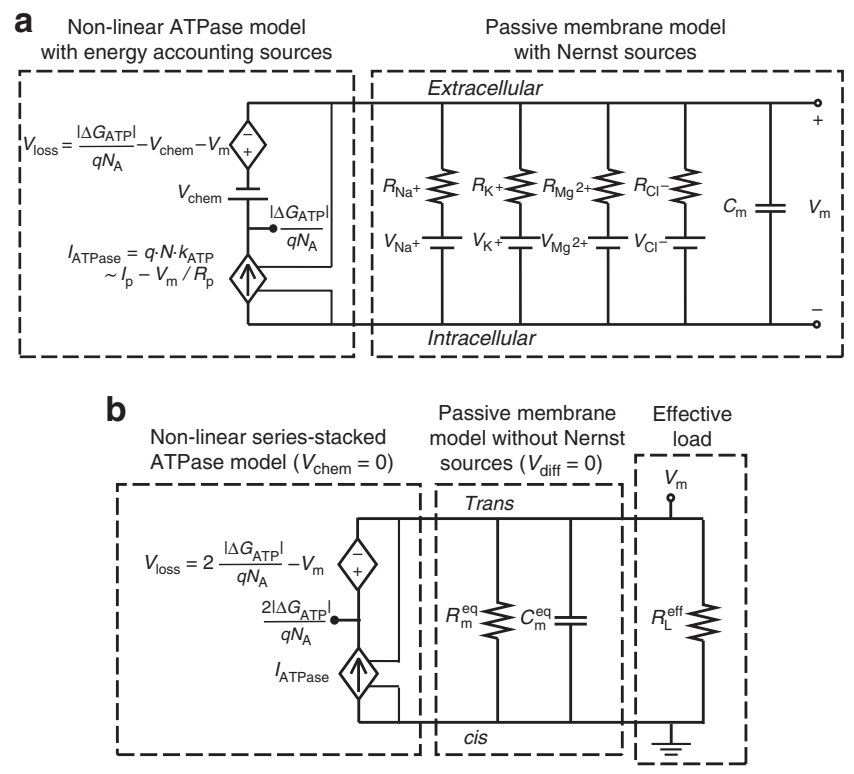

Figure 4 | Equivalent circuit models. (a) Single-biocell equivalent circuit model with additional voltage sources to account for energy delivered by the pump. (b) Stacked membrane equivalent circuit model.

ATP hydrolysis reaction per mole of ATP at given buffer conditions and $N_{\mathrm{A}}$ is Avogadro's number. Since every charge that passes through $I_{\mathrm{ATPase}}$ corresponds to a single hydrolysis event, we can use two voltage sources in series with $I_{\text {ATPase }}$ to 
independently account for the energy expended by the pumps both in moving charge across the electric potential difference and in moving ions across the chemical potential difference. The dependent voltage source $V_{\text {loss }}$ in this branch fixes the voltage across $I_{\mathrm{ATPase}}$, and the total power produced by the pump current source is $\left(\left|\Delta G_{\mathrm{ATP}}\right| / N_{\mathrm{A}}\right)\left(N k_{\mathrm{ATP}}\right)$, which is the product of the energy released per molecule of ATP, the number of active ATPases and the ATP turnover rate. The power dissipated in voltage source $V_{\text {chem }}$ models the work performed by the ATPases in transporting ions against a concentration gradient. In the case of the $\mathrm{Na}^{+} / \mathrm{K}^{+}$ATPase, $V_{\text {chem }}$ is given by $3 V_{\mathrm{Na}^{+}}+2 V_{\mathrm{K}^{+}}$. The power dissipated in this source is introduced back into the circuit in the power generated by the Nernst independent voltage sources, $V_{\mathrm{Na}^{+}}$and $V_{\mathrm{K}^{+}}$. The power dissipated in the dependent voltage source $V_{\text {loss }}$ models any additional power not used to perform chemical or electrical work.

In our system, no significant ion concentration gradient exists, $V_{\text {chem }}=0$, and the expanded membrane model of Fig. 4a becomes the passive membrane model of Fig. $4 \mathrm{~b}$. The efficiency of the biocell is given by $\eta_{\text {biocell }}=P_{R_{\mathrm{L}}^{\text {eff }}} / P_{\mathrm{ATP}}=V_{\mathrm{m}}^{2} / R_{\mathrm{L}}^{\text {eff }} /\left[\left(2 \frac{\left|\Delta G_{\mathrm{ATP}}\right|}{q N_{\mathrm{A}}}\right)\right.$ $\left.\left(I_{\mathrm{p}}-\frac{V_{\mathrm{m}}}{R_{\mathrm{p}}}\right)\right]$ with $V_{\text {loss }}=2 \frac{\left|\Delta G_{\mathrm{ATP}}\right|}{q N_{\mathrm{A}}}-V_{\mathrm{m}}$ (the factor of two arises from the series stack, see Supplementary Discussion). The

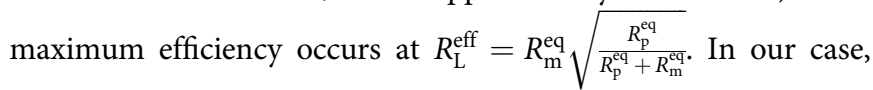
$\eta_{\text {biocell }} \sim \eta_{\text {biocell }}^{\max }=14.9 \%$ and the overall energy efficiency of the system $\eta=\eta_{\text {converter }} \times \eta_{\text {biocell }}=8.84 \%$.

Higher membrane potentials can be sustained in systems with electrogenic ion pumps engineered with lower $V_{\text {chem. }}$. For example, proton-pumping ATPases, such as those found in the plasma membrane of the yeast Schizosaccharomyces pombe, transport only a single proton per hydrolysed molecule of ATP. Less chemical work is performed $\left(V_{\text {chem }}=14 \mathrm{mV}\right.$, for $\Delta \mathrm{pH}=0.1$ ) compared with the sodium-potassium pumps. As a result, these pumps can sustain a membrane potential of up to $280 \mathrm{mV}^{19,20}$. In our system, with a similar number of active incorporated pumps and $R_{\mathrm{m}}$ in excess of $575 \mathrm{G} \Omega$ due to the absence of other channels, voltages of $227 \mathrm{mV}$ could have been achieved with these pumps.

Living systems use ATP much more efficiently than in the in vitro system developed here because $V_{\text {loss }}$ is considerably smaller in living systems due to the presence of a non-zero $V_{\text {chem }}$. Under typical physiological conditions, $V_{\text {chem }}$ is typically on the order of $336.5 \mathrm{mV}$ at $20^{\circ} \mathrm{C}$ (ref. 21) with all power lost in $V_{\text {chem }}$ converted to electrical power by the voltage sources representing the Nernst potentials of the cell.

\section{Discussion}

Integration of ATP-harvesting ion pumps could provide a means to power future CMOS microsystems scaled to the level of individual cells $\mathrm{s}^{22}$. In molecular diagnostics, the integration of pore-forming proteins such as alpha haemolysin ${ }^{23}$ or MspA porin $^{24}$ with CMOS electronics is already finding application in DNA sequencing 25 . Exploiting the large diversity of function available in transmembrane proteins in these hybrid systems could, for example, lead to highly specific sensing platforms for airborne odorants or soluble molecular entities ${ }^{26,27}$. Heavily multiplexed platforms could become high-throughput in vitro drug-screening platforms against this diversity of function. In addition, integration of transmembrane proteins with CMOS may become a convenient alternative to fluorescence for coupling to synthetic biological systems ${ }^{28}$.

\section{Methods}

Teflon cell preparation and lipid bilayer formation. The $250-\mu \mathrm{m}$ delrin pore is seated in a Teflon cell, washed with $0.1 \% \mathrm{HCl}, 40 \mathrm{mM}$ trisodium phosphate (TSP), rinsed with deionized water, and dried with $\mathrm{N}_{2}$. The pore is pretreated with $1 \mu \mathrm{l}$ of DOPC lipids (Avanti Polar lipids) dissolved in $n$-hexane $\left(5 \mathrm{mg} \mathrm{ml}^{-1}\right)$. The hexane is evaporated in a high-vacuum desiccator for $10 \mathrm{~min}$. The Teflon cell is fixed to the chip package (bond wires are protected by donut epoxy encapsulation) and the cis and trans chambers are filled with experiment buffer $(50 \mathrm{mM} \mathrm{NaCl}, 10 \mathrm{mM} \mathrm{KCl}$, $3 \mathrm{mM} \mathrm{MgCl}_{2}, 10 \mathrm{mM}$ HEPES, pH7.3). A cleaned and equilibrated $\mathrm{Ag} / \mathrm{AgCl}$ pellet electrode is inserted into the cis chamber (ground). The electrode resistance and solution resistance are measured by applying a $500-\mathrm{ms} 10-\mathrm{mV}$ voltage step and averaging the current of the last $5 \mathrm{~ms}$ of the step response after the charging current transient has decayed away. The electrical resistance is verified to be $<20 \mathrm{k} \Omega$ (typically $15 \mathrm{k} \Omega$ ), and the open circuit DC voltage offset between electrodes is confirmed to be $<3 \mathrm{mV}$. If either of these conditions is not met, the electrodes are cleaned as described below.

The lipid bilayer is formed by dipping a plastic pipette tip into a solution of $n$-decane containing DOPC $\left(20 \mathrm{mg} \mathrm{ml}^{-1}\right)$, forming an air bubble by depressing the pipette plunger under the surface of the buffer in the cis chamber and touching the air bubble to the pore. After $1 \mathrm{~min}$, the bilayer capacitance is extracted by applying a voltage triangle wave and monitoring the resulting current square wave, $C_{\mathrm{m}}=\frac{I_{\mathrm{c}-\max }}{\Delta V_{\mathrm{m}} / \Delta t^{\circ}}$. Bilayers with capacitance $<150 \mathrm{pF}$ are reformed by touching another air bubble to the pore. The bilayer resistance is extracted by applying a series of 16 10 -s square-wave voltages from -50 to $100 \mathrm{mV}$ in $10 \mathrm{mV}$ increments while monitoring the current. The final $5 \mathrm{~s}$ of each step are averaged after chargingcurrent transients have decayed away. The resistance is determined to be the inverse of the slope of a linear fit. Bilayers with resistances $<300 \mathrm{G} \Omega$ are reformed. We observe that if a successful bilayer cannot be formed within five tries, successive attempts are also likely to be unsuccessful, suggesting that the likelihood of formation of a high resistance stable bilayer is dependent on pore preparation (cleaning and pre-treatment). This is important as the bilayer is reformed many times once the process of ATPase incorporation begins. All measurements are performed in a Faraday cage.

Electrode preparation. Two identical $\mathrm{Ag} / \mathrm{AgCl}$ pellet electrodes (Warner Instruments) are used for biocell characterization experiments. Pellet electrodes are shorted together at the wire end for the duration of preparation. The electrodes are immersed in $0.1 \% \mathrm{HCl}$ for $1 \mathrm{~min}$. The electrodes are rinsed under a DI water stream and immersed in $3 \%$ sodium hypochlorite for $30 \mathrm{~min}$. The electrodes are rinsed again under a DI water stream and immersed in experiment buffer $(50 \mathrm{mM}$ $\mathrm{NaCl}, 10 \mathrm{mM} \mathrm{KCl}, 3 \mathrm{mM} \mathrm{MgCl}_{2}, 10 \mathrm{mM}$ HEPES, pH 7.3) until use. Concentrations employed are very close to physiological conditions, except for the lack of an ion gradient across the membrane. The electrode preparation protocol is repeated if the open-circuit potential offset is found to be above $3 \mathrm{mV}$. The electrode offsets are verified at the end of each experiment to be $<5 \mathrm{mV}$.

For the IC experiments, the top surface of the IC that is coated with $500 \mathrm{~nm}$ of silver by electron-beam evaporation and chloridized with $3 \%$ sodium hypochlorite for 30 min serves as the trans chamber electrode (working electrode, WE). An $\mathrm{Ag} / \mathrm{AgCl}$ pellet electrode (counter electrode) is inserted into the cis chamber as with the characterization experiments and attached to circuit ground on the board. Cleaning and equilibration of the electrodes are carried out as above; however, the chip is placed in a socket with access to the 'wire' end of the planar electrode through a pin on the board. A Teflon chamber attached by silicone epoxy (Kwik-cast) to the surface of the chip package serves as a reservoir for the above solutions. It is necessary to momentarily disconnect the 'wire' ends of the electrodes for the DI water rinse. Electrode offsets exceeding $3 \mathrm{mV}$ are observed more frequently when using the planar electrode; however, we find that this is easily remedied by increasing the chloridization time to $1 \mathrm{~h}$.

Proteoliposome preparation. Hyperosmotic proteoliposomes to be fused with the planar bilayers are prepared using a modified version of the dual-detergent protocol in ref. 29, which reported that the use of dual detergents yields vesicles with higher transport activity (40-60\% of original enzyme activity) than formation using either detergent alone. DOPC dissolved in chloroform (Avanti Polar Lipids) are transferred to a round-bottom flask using a glass syringe (Hamilton). Chloroform is evaporated under high vacuum on a Buchi Rotavap for $5 \mathrm{~min}$. The flask is transferred to a high-vacuum desiccator for a minimum of $2 \mathrm{~h}$ to aid in removal of residual solvent. Vesicle buffer containing ( $575 \mathrm{mM} \mathrm{NaCl}, 5 \mathrm{mM}$ HEPES, $\mathrm{pH} 7.3$ ) is added to the flask (final lipid concentration of $4 \mathrm{mg} \mathrm{ml}^{-1}$ ), and the flask is placed on a vortexer (2,500 r.p.m.) for $10 \mathrm{~min}$. Lipid vesicles are mixed with $n$-octyl- $\beta$-Dglucopyranoside ( $n$-OG; detergent:lipid weight ratio 3:1) and placed on a rotator for $5 \mathrm{~min}$ until the vesicle solution become clear. A solution of $n$-decyl- $\beta$-Dmaltopyranoside dissolved in vesicle buffer is added to purified ATPase (detergent:protein weight ratio 3:1) and the tube is gently rotated for $1 \mathrm{~min}$. Purified ATPase lyophilized powder contains 90\% sucrose and 0.4\% EDTA. Solubilized vesicles and solubilized ATPase (lipid:protein weight ratio 5:1) are combined in 2-ml centrifuge tubes (total volume of solution $270 \mu \mathrm{l}$ ) and rotated for $1 \mathrm{~min}$. A volume of pre-equilibrated Bio-Beads SM-2 (Bio-Rad) equal to the volume of lipid/protein mixture is added to the tubes and the tubes are placed back on the rotator. After $30 \mathrm{~min}$ the tubes are filled to the top with Bio-Beads and rotated for $2 \mathrm{~h}$. Proteoliposomes are removed from Bio-Beads and used immediately. 
Electrical measurement methods. Voltage-clamp (current) measurements are performed using a HEKA EPC10 bench-top patch clamp amplifier. The headstage gain is set to $500 \mathrm{G} \Omega$. Two series programmable analog filters are used in all current measurements.

Filter 1: analog 3-pole Bessel, $f_{\mathrm{c}}=10 \mathrm{kHz}$.

Filter 2: analog 4-pole Bessel $f_{\mathrm{c}}=100 \mathrm{~Hz}$.

For $I-V$ measurements, the last $5 \mathrm{~s}$ of a $10-\mathrm{s}$ measurement are averaged.

Patch-clamp amplifiers typically employ large junction gate field-effect transistor input transistors to minimize noise. As a result, the amplifier has large input bias and input offset currents, on the order of a few hundred femtoamps. To compensate for these currents, once a linear fit is applied to the $I-V$ sweep of a bilayer without ATPases, the measured short-circuit current is subtracted from all data points, such that the curve passes through the origin.

The input resistance, $R_{\mathrm{in}}$, of the benchtop amplifier is $100 \mathrm{G} \Omega$, which shunts the membrane resistance. This shunt resistance compromises the measurement and instead we use a custom two-stage amplifier using a LMC6041 opamp in noninverting unity gain feedback followed by an ADA4661 opamp in non-inverting configuration with a gain of $5 \mathrm{~V} / \mathrm{V}$. Short time-scale measurements $(<1 \mathrm{~min})$ are acquired using an Agilent DSO7054A oscilloscope. Measurements exceeding 1 min are acquired using an Keithley 2182A nanovoltmeter.

\section{References}

1. Rottenberg, $\mathrm{H}$. The measurement of membrane potential and deltapH in cells, organelles, and vesicles. Methods Enzymol. 55, 547-569 (1979).

2. Himes, C., Carlson, E., Ricchiuti, R. J., Otis, B. P. \& Parviz, B. A. Ultralow voltage nanoelectronics powered directly, and solely, from a tree. IEEE Trans. Nanotechnol. 9, 2-5 (2010).

3. Mercier, P. P., Lysaght, A. C., Bandyopadhyay, S., Chandrakasan, A. P. \& Stankovic, K. M. Energy extraction from the biologic battery in the inner ear. Nat. Biotechnol. 30, 1240-1243 (2012).

4. Halámková, L. et al. Implanted Biofuel Cell Operating in a Living Snail. J. Am. Chem. Soc. 134, 5040-5043 (2012).

5. Briskin, D. P., Basu, S. \& Assmann, S. M. Characterization of the Red Beet Plasma Membrane H +-ATPase Reconstituted in a Planar Bilayer System. Plant Physiol. 108, 393-398 (1995).

6. Hirata, H., Ohno, K., Sone, N., Kagawa, Y. \& Hamamoto, T. Direct measurement of the electrogenicity of the $\mathrm{H}+$-ATPase from thermophilic bacterium PS3 reconstituted in planar phospholipid bilayers. J. Biol. Chem. 261, 9839-9843 (1986)

7. Seifert, K., Fendler, K. \& Bamberg, E. Charge transport by ion translocating membrane proteins on solid supported membranes. Biophys. J. 64, 384-391 (1993).

8. Naumann, R. et al. Coupling of proton translocation through ATPase incorporated into supported lipid bilayers to an electrochemical process. Bioelectrochem. Bioenerg. 42, 241-247 (1997).

9. Ikematsu, M., Sugiyama, Y., Iseki, M., Muneyuki, E. \& Mizukami, A. Direct reconstitution of bacteriorhodopsin into planar phospholipid bilayers-detergent effect. Biophys. Chem. 54, 155-164 (1995).

10. Horn, C. \& Steinem, C. Photocurrents generated by bacteriorhodopsin adsorbed on nano-black lipid membranes. Biophys. J. 89, 1046-1054 (2005).

11. Bamberg, E. et al. Photocurrents generated by bacteriorhodopsin on planar bilayer membranes. Biophys. Struct. Mechanism 5, 277-292 (1979).

12. Braun, C. J., Baer, T., Moroni, A. \& Thiel, G. Pseudo painting/air bubble technique for planar lipid bilayers. J. Neurosci. Methods 233, 13-17 (2014).

13. Kabakov, A. Y. Activation of KATP channels by $\mathrm{Na} / \mathrm{K}$ pump in isolated cardiac myocytes and giant membrane patches. Biophys. J. 75, 2858-2867 (1998).

14. Landowne, D. \& Ritchie, J. M. The binding of tritiated ouabain to mammalian non-myelinated nerve fibres. J. Physiol. 207, 529-537 (1970).

15. Glitsch, H. G. Electrophysiology of the sodium-potassium-ATPase in cardiac cells. Physiol. Rev. 81, 1791-1826 (2001).

16. Gadsby, D. C., Kimura, J. \& Noma, A. Voltage dependence of Na/K pump current in isolated heart cells. Nature 315, 63-65 (1985)

17. Demkov, A. A. \& Navrotsky, A. Materials Fundamentals of Gate Dielectrics Vol. 9 (Springer, 2005).
18. Zhirnov, V. V. \& Cavin, III R. K. Microsystems for Bioelectronics: the Nanomorphic Cell (William Andrew, 2010).

19. Imai, T. \& Ohno, T. Measurement of yeast intracellular $\mathrm{pH}$ by image processing and the change it undergoes during growth phase. J. Biotechnol. 38, 165-172 (1995).

20. Ziegler, W., Slayman, C. L. \& Cartwright, C. P. Reconstitution of a plasmamembrane $\mathrm{H}(+)$-ATPase into bilayer lipid membrane. Gen. Physiol. Biophys. 12, 429-443 (1993).

21. Kuffler, S. W., Nicholls, J. G. \& Martin, A. R. From Neuron to Brain: A Cellular Approach to the Function of the Nervous System (Sinauer, 1984).

22. Choi, J. et al. Matching the power, voltage, and size of biological systems: a $\mathrm{nW}$-scale, $0.023-\mathrm{mm}^{3}$ pulsed $33-\mathrm{GHz}$ radio transmitter operating from a 5kT/q-supply voltage. IEEE Trans. Circuits Syst. 62, 1950-1958 (2015).

23. Kasianowicz, J. J., Brandin, E., Branton, D. \& Deamer, D. W. Characterization of individual polynucleotide molecules using a membrane channel. Proc. Nat Acad. Sci. USA 93, 13770-13773 (1996).

24. Butler, T. Z., Pavlenok, M., Derrington, I. M., Niederweis, M. \& Gundlach, J. H. Single-molecule DNA detection with an engineered MspA protein nanopore. Proc. Natl Acad. Sci. 105, 20647-20652 (2008).

25. Maitra, R. D., Kim, J. \& Dunbar, W. B. Recent advances in nanopore sequencing. Electrophoresis 33, 3418-3428 (2012).

26. Zhang, X. \& Firestein, S. The olfactory receptor gene superfamily of the mouse Nat. Neurosci. 5, 124 (2002).

27. Saito, H., Chi, Q., Zhuang, H., Matsunami, H. \& Mainland, J. D. Odor coding by a Mammalian receptor repertoire. Sci. Signal 2, ra9 (2009).

28. Voigt, C. A. Synthetic biology: bacteria collaborate to sense arsenic. Nature 481, 33-34 (2012).

29. Alpes, H., Apell, H. J., Knoll, G., Plattner, H. \& Riek, R. Reconstitution of $\mathrm{Na}+/ \mathrm{K}+$-ATPase into phosphatidylcholine vesicles by dialysis of nonionic alkyl maltoside detergents. Biochim. Biophys. Acta 946, 379-388 (1988).

30. White, S. H. A study of lipid bilayer membrane stability using precise measurements of specific capacitance. Biophys. J. 10, 1127-1148 (1970).

\section{Acknowledgements}

We thank Lance Kam for discussions and Trevor Finney for help with artwork. This work was supported in part by the Keck Foundation, by the Office of Naval Research under Grant N00014-13-1-0375, and by the National Institutes of Health under Grant 5R01HG006879.

\section{Author contributions}

J.M.R., J.L., S.R., J.K.R. and K.S. designed the architecture. J.M.R. designed the microchip, performed the experiments and analysed data. All authors discussed the results, and J.M.R. and K.S. wrote the paper.

\section{Additional information}

Supplementary Information accompanies this paper at http://www.nature.com/ naturecommunications

Competing financial interests: The authors declare no competing financial interests.

Reprints and permission information is available online at http://npg.nature.com/ reprintsandpermissions/

How to cite this article: Roseman, J. M. et al. Hybrid integrated biological-solid-state system powered with adenosine triphosphate. Nat. Commun. 6:10070 doi: 10.1038/ ncomms10070 (2015)

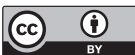

This work is licensed under a Creative Commons Attribution 4.0 International License. The images or other third party material in this article are included in the article's Creative Commons license, unless indicated otherwise in the credit line; if the material is not included under the Creative Commons license, users will need to obtain permission from the license holder to reproduce the material. To view a copy of this license, visit http://creativecommons.org/licenses/by/4.0/ 